\title{
Peripheral Arterial Disease in Patients with Atrial Fibrillation
}

\author{
Zinkienė D*, Mašanauskienė E, Naudžiūnas A and Sadauskas S \\ Lithuanian University of Health Sciences, Medical Academy, Department of Internal Diseases, Lithuania
}

*Corresponding author: Zinkienė D, Assistant lecturer, Lithuanian University of Health Sciences, Medical Academy, Department of Internal Diseases, Lithuania

\section{ARTICLE INFO}

Received: 幽 January 29, 2019

Published: 慧 February 05, 2019

\section{ABSTRACT}

Citation: Zinkienė D, Mašanauskienė E, Naudžiūnas A, Sadauskas S. Peripheral Arterial Disease in Patients with Atrial Fibrillation. Biomed J Sci \& Tech Res 14(1)-2019. BJSTR. MS.ID.002491.

\section{Mini Review}

Peripheral Arterial Disease (PAD) is defined as a systemic atherosclerotic disease, which occurs due to narrowing and obstruction of major systemic arteries other than coronary arteries and aorta. It affects 200 million individuals worldwide and is independent risk factor for total mortality and incident cardiovascular or cerebrovascular events [1]. Da-ta of Rotterdam study present that only 10\% of 55-59 years old participants had PAD and $60 \%$ subjects in age group $>85$ years was diagnosed with this condition.

According to the National Health and Nutrition Examination Survey 1999-2000 major risk factors for PAD include arterial hypertension, diabetes, hypercholesterolemia, smoking, obesity. Over $95 \%$ of patients with peripheral arterial disease has one of these risk factors and over $70 \%$ has two major risk factors. Some studies suggest that chronic renal failure may influence manifestation of PAD. Collaborative meta-analysis of international cohorts made by Matsushita et al. showed that even mild-tomoderate chronic kidney disease increases risk of incident peripheral artery disease [2].

Increased inflammatory markers such as C-Reactive Protein (CRP) have an impact on PAD. CRP demonstrated a statistically significant correlation of risk for PAD compared with patients without PAD [3-5]. Classical clinical manifestation of low extremities PAD include aching pain especially in the calves which occurs during activity and reliefs by rest (intermittent claudication), poikilothermic (cold sensation), pallor of the limb and ulceration. Most of the cases patients are associating these signs with other earlier diagnosed disease such as diabetes, arthritis, etc. In the PIPETTE study $33 \%$ of participants with severe symptoms of intermittent claudication that warranted subsequent endovascular intervention had not previously presented to their general practice [3]. Up to $50 \%$ of patients with PAD has no symptoms and this group is most under-recognised and under-treated. This is one of the reasons why PAD is diagnosed in severe stages with high mortality risk.

Early diagnosis of PAD is important to prevent cardiovascular complications. Ac-cording to NHANES mortality from cardiovascular events among patients diagnosed with PAD was 29.2\% [4]. Simple, non-invasive, fast and cheap tool to diagnose this condition is the Ankle-Brachial Index (ABI). The measurement of blood pressure at the ankle was proposed as a test for PAD as early as 1950 and led to the development of the Ankle-Brachial Index (ABI) [1]. ABI is defined as the ratio between systolic blood pressure at the ankle and in the arm. Measurements may be obtained using Doppler ultrasound or oscillometric sphygmomanometry. An ABI of $<0.9$ is considered as abnormal and is associated with symptomatic and asymptomatic forms of PAD and higher risk of cardiovascular events. According to 2017 ECS guidelines on the diagnosis and treatment of peripheral arterial disease an $\mathrm{ABI}<0.90$ is associated with 2- to 3-fold increased risk of total and cardiovascular death.

American Heart Association recommends determining anklebrachial index in the following cases: adults $\geq 65$ years old, adults $\geq 50$ years old with a history of smoking or diabetes, and adults of any age with exertional leg symptoms or non-healing wounds. European Society of Cardiology suggests considering screening with $\mathrm{ABI}$ in patients with Coronary Artery Disease (CAD).

ARTPER cohort study showed that at 9 years $20 \%$ of patients with low ABI had ma-jor cardiovascular events such as myocardial 
infarction, ischaemic stroke and vascular mortality. Patients with borderline ABI (0.90-0.99) should be considered also as a high-risk group and further diagnostic tests should be made [6]. The MultiEthnic Study of Atherosclerosis (6570 participants) identified that patient with PAD and low ABI $(<0.90)$ had twice risk for the development of atrial fibrillation or stroke [7]. Peripheral arterial disease is common in patients with Atrial Fibrillation (AF), but the association between these two conditions are not completely understood. Both diseases share risk factors such as diabetes, arterial hypertension, hypercholesterolemia and are associated with increased levels of inflammation and platelet-mediated thrombosis [7].

Cohort study (5143 participants) reporter that incident rate of AF significantly higher in group with PAD compared with patients without PAD. Risk of AF increases 6\% with each 0.1 decreasing of $\mathrm{ABI}[8,9]$. Other large cohort study $(582,635$ subjects) resulted $\mathrm{PAD}$ as independent risk factor for AF - for 10 years follow-up period events of AF was significantly higher for participants with PAD, they had 1.286 times higher incident rate to develop AF [10]. In the ARAPACIS study $21 \%$ of patients with Non-Valvular Atrial Fibrillation (NVAF) had ABI $<=0.90$, which indicates that NVAF is associated with systemic atherosclerosis, including PAD [9].

In other hand several studies suggest that increased heart rate in $\mathrm{AF}$ patients has major impact for arterial stiffness and may affect measurements of ABI [11,12]. In general, the ankle-brachial index determines how well blood is slowing in body. During atrial fibrillation peripheral pulse wave may not be registered correctly, which can lead to an incorrect $A B I$ result. We know that risk factors of AF such as diabetes, hypertension are associated with inflammation. Atherosclerosis is also a chronic inflammatory disease, so this condition may result falsely high ratio due to the presence of incompressible calcified blood vessels.

E. Mašanauskienė et al. [13] suggested an alternative noninvasive method to diagnose PAD - Impedance Plethysmography (IP). Extremities impedance results about peripheral vascular response to the quantity and velocity of circulating blood and peripheral vascular resistance. Significant correlation were found between ABI and IP parameter Crest Time (CT). They found CT sensitivity of $73 \%$ and specificity of $95 \%$. Dachun Xu et al. [14] reported specificity (83.3-99.0\%) and accuracy (72.1-89.2\%) of $\mathrm{ABI}$ by diagnosing $\geq 50 \%$ stenosis, but sensitivity ranged from 15 to $79 \%$ especially elderly participants or subjects with diabetes.
Considering to all results impedance plethysmography could be used to diagnose peripheral circulation disorders, especially in patients with high risk of atherosclerosis and other conditions such as atrial fibrillation which may lead to falsely ABI results.

\section{References}

1. Michael H Criqui, Victor Aboyans (2015) Epidemiology of peripheral artery disease. Circ Res 116(9): 1509-1526.

2. Matsushita K, Ballew SH, Coresh J, Arima H, Ärnlöv J, et al. (2017) Measures of chronic kidney disease and risk of incident peripheral artery disease: A collaborative meta-analysis of individual participant data. Lancet Diabetes Endocrinol 68(2): 145-150.

3. Jane H Davies, Jonathan Richards, Kevin Conway, Joyce E Kenkre, Jane EA Lewis et al. (2017) Primary care screening for peripheral arterial disease: A cross-sectional observational study. Br J Gen Pract 67(655): e103-e110.

4. Stephen M Amrock, Cherrie Z Abraham, Enjae Jung, Pamela B Morris, Michael D Shapiro (2017) Risk factors for mortality among individuals with peripheral arterial disease. Am J Cardiol 120(5): 862-867.

5. Patrick A Stonen, Michael Yacoub (2014) Inflammatory biomarkers in peripheral arterial disease. Semin Vasc Surg 27(2014): 148-151.

6. Teresa Alzamora, Rosa Forés, Guillem Pera, José Miguel Baena-Díez, et al. (2019) Low, borderline and normal ankle-brachial index as a predictor of incidents outcomes in the Mediterranean based-population ARTPER cohort after 9 years follow-up. PLoS ONE 14(1): e0209163.

7. O'Neal WT, Efird JT, Nazarian S, Alonso A, Heckbert SR, et al. (2014) Peripheral arterial disease and risk of atrial fibrillation and stroke: The multi-ethnic study of atherosclerosis. J Am Heart Assoc 3(6): e001270.

8. Griffin WF, Salahuddin T, O’Neal WT, Soliman EZ (2016) Peripheral arterial disease is associated with an increased risk of atrial fibrillation in the elderly. Europace 18(6): 794-798.

9. Violi F, Daví G, Hiatt W, Lip GY, Corazza GR, et al. Prevalence of peripheral artery disease by abnormal ankle-brachial index in atrial fibrillation: implications for risk and therapy. J Am Coll Cardiol 63(14): 1456-1457.

10. Yu-Sheng Lin, Tao-Hsin Tung, Jui Wang, Yu-Fen Chen, Tien-Hsing Chen, et al. (2016) Peripheral arterial disease and atrial fibrillation and risk of stroke, heart failure hospitalization and cardiovascular death: A nationwide cohort study. Int J Cardiol 203: 204-211.

11. Chu CY, Lin TH, Hsu PC, Lee WH, Lee HH, et al. (2013) Heart rate significantly influences the relationship between atrial fibrillation and arterial stiffness. Int J Med Sci 10(10): 1295-1300.

12. Byoung-Jin Parka, Hye-Ree Lee, Jae-Yong Shim, Jung-Hyun Lee, DongHyuk Jung, et al. Association between resting heart rate and arterial stiffness in Korean adults. Arch Cardiovasc Dis 103(4): 246-252.

13. Mašanauskienè E, Sadauskas S, Naudžiūnas A, Unikauskas A, Stankevičius E (2014) Impedance plethysmography as an alternative method for the diagnosis of peripheral arterial disease. Medicina (Kaunas) 50(6): 334-339.

14. Xu D, Li J, Zou L, Xu Y, Hu D, et al. (2010) Sensitivity and specificity of the ankle-brachial index to diagnose peripheral artery disease: A structured review. Vasc Med 15(5): 361-369. 


\section{ISSN: 2574-1241}

DOI: 10.26717.BJSTR.2019.14.002491

Zinkienẻ D.Biomed J Sci \& Tech Res

CC (i) This work is licensed under Creative

Submission Link: https://biomedres.us/submit-manuscript.php

$\begin{array}{ll}\text { BIOMEDICAL } & \text { Assets of Publishing with us } \\ \text { RESEARCHES } & \text { - Global archiving of articles } \\ & \text { - Immediate, unrestricted online access } \\ & \text { - Rigorous Peer Review Process } \\ \end{array}$

